\title{
我国数字广播的发展方向
}

\author{
王国裕, 张红升", 陆明莹 \\ 重庆邮电大学微电子工程重点实验室, 重庆 400065 \\ * 联系人, E-mail: zhanghs@ cqupt.edu.cn
}

2014-02-11 收稿, 2014-04-16 接受

重庆市教委重点实验室建设计划和科研项目(KJ130530)、2013 重庆高校创新团队建设计划、重庆市科委基础与前沿研究项目 (2013JCYJA40006)、重庆市科技人才培养计划(cstc2013kjrc-tdjs40009)和江苏省科技项目(BC2011079)资助

\begin{abstract}
摘要 数字广播的发展方向从传统的音频节目转向公众化多媒体信息发布，这将使得广播 技术的本征优势得以发挥，并能弥补通讯和互联网技术的不足，从而使广播技术成为现代通 信技术的互补技术而得到长足发展。本文综述了数字广播 DAB 技术在公共信息发布和教 育、预警救灾、景区安全等专业领域的应用，显示了公众化应用巨大的市场潜力; 分析了我 国发展 DAB 所面临的困难，而公众化应用是走出困境的方向; 进而介绍了我国在 DAB 技术 和公众化应用方面所具备的核心技术和创新性成果, 说明我国可以在这个领域领先于国际 水平, 并形成一个特色产业.
\end{abstract}

关键词
数字广播
数字音频广播
数字多媒体广播
公众化应用
预警
应急救灾
景区安全

但是总体来说, 数字广播发展比预期慢很多, 其 主要原因是在广播数字化的定位上有很大局限性, 仅以代替模拟广播为目的. 在当今信息时代, 通信技 术飞速发展的背景下, 单一的广播节目内容即使数 字化也难免被边缘化. 而我国除了应用的单一性, 政 策的局限性也是重要因素之一, 使得 DAB 或其他数 字广播技术的发展举步维艰. 实际上, 数字广播的本 征特性使其在受众性、可靠性等方面比通信技术(通 讯和网络等)更具优越性, 还可弥补通信技术的不足, 能够在通信网络不能胜任甚至痽疾时发挥独特的作 用. 当数字广播以此来重新定位时, 将会发现其独特 的优势, 可以在语音广播节目之外创造出许多新的 应用和产业方向, 且在新的形势下更具有生命力和 发展空间. 在通信技术迅猛发展的巨大压力下，广播 技术依靠自身的优点, 能够化压力为动力, 至少可以 成为通讯和互联网的互补而显示其价值.

本文分析了数字广播的本征特性、通信技术的不 足和广播技术的互补作用, 以及我国发展 DAB 数字 广播所面临的困难和政策性误区; 在此基础上提出

引用格式: 王国裕, 张红升, 陆明䒯. 我国数字广播的发展方向. 科学通报, 2014, 59: 2320-2327 Wang G Y, Zhang H S, Lu M Y. Developmental trend of digital broadcasting in China (in Chinese). Chin Sci Bull (Chin Ver), 2014, 59: 23202327, doi: 10.1360/N972014-00081 
了数字广播公众化应用的发展方向, 讨论了 $\mathrm{DAB}$ 在 教育、预警救灾、景区安全等专业领域的应用; 进而 介绍了我国在数字广播技术和公众化应用方面所具 备的核心技术和创新性成果, 说明我国可以在此领 域领先于国际水平, 并形成一个新的产业. 本文关于 数字广播的技术特征、政策和发展方向的论述, 希望 对广电行业和政策/标准制定有参考价值; 所提出的 公众化应用的市场前景希望受到研发人员和产业界 关注; 其在教育和公共信息发布、公共安全领域良好 的应用效果希望受到政府相关部门的重视和青睐. 当通信技术一味追求更高速度从而使得系统越来越 复杂的同时，广播技术所具有的简单、实用、经济和 可靠的特性恰恰展示了另一个技术发展的方向.

\section{1 技术和政策分析}

\section{1 广播技术和通信技术的比较}

广播与通信的技术特征区别可以概括为两点: 广播的“一对全体(one to all)”和通信的“一对部分(one to some)”; 广播的单向性和通信的双工性.

所谓“一对全体”是指广播的一个发射台覆盖区 域内所有终端, 可以同时接收到发射台播发的节目 内容. 而通信技术 “一对部分” 是在一个基站或服务 器覆盖区域内, 只能服务部分用户. 因而移动通讯和 互联网同时连通的用户数是有限的，而广播可同时 接收的用户则是无限的. 当大批用户同时访问互联 网或通信网时, 服务器或基站就会堵塞, 导致谁也无 法连接. “一对全体”是广播技术最重要的特征和优势, 不仅突破了用户数的限制, 而且传送信息的成本大 为降低, 特别在用于公共信息发布时, 广播是最经 济、最快捷、最绿色环保的技术手段.

通信的双工成就了“双向”通讯和互联网之“互”. 相比之下，广播的单向性限制了许多应用. 但广播的 单向性在公共信息发布和公众化应用方面恰恰又避 免了双工所带来的用户的选择权和竞争、垃圾信息、 黑客人侵、网络安全等一系列负面问题. 也正因为双 工性使得通信传输信息的效率低(一对一), 并常有 断、卡和延迟现象, 这使得通信平台由于接收质量不 能保证而不适宜用于诸如教学等重要场合.

除了技术特征以外, 还必须提及系统可靠性. 广 播系统的一大优势是结构简单、容易维护、抗灾能力 强. 而通信系统由于系统复杂, 使其很容易被影响,
如在重大灾害中，通信网络往往先被损坏，而这时是 最需要它们发挥作用的时候，因此通信网络是“平时 离不开, 关键时候靠不住”.

分析通信系统的复杂性根源, 有其为了实现双 工性所付出的代价, 也因为一味追求更高速度使得 系统越来越复杂. 实际上, 当通讯系统愈来愈多用于 信息获取时，应当从源头上考虑单向性和双工性的 结合，从降低数据流量着手. 作者希望通过本文的介 绍，能够使得广播用于公共信息发布的思路和实践 对于通信行业有所启示.

\section{2 个性化与公众化定位}

在当今信息化时代, 电子产品层出不穷, 无论是 手机、电脑、视听产品, 都以时尚和个性化为卖点. 数 字收音机也在跟随这个路线 ${ }^{[5 \sim 8]}$. 单一的广播节目内 容使得数字收音机的市场规模与通信产品相比越来 越萎缩. 另一方面, 个性化电子产品的繁荣倒使得公 众化功能在退化. 尽管每人都有手机，都上网，但通 过手机和网络发送重要信息的效果并不好. 近年来 灾害频发并累累造成重大伤亡. 造成这种现象的原 因之一就是预警和应急通信技术过于依赖通信网和 互联网, 但两者不能胜任重大灾害的及时预警和紧 急情况下应急指挥 ${ }^{[9 \sim 11]}$.

其实在信息化时代之前，面向公众的信息发布 早已不是问题，比如二十世纪五六十年代的有线广 播和遍及城乡的“大喇叭”，使得“北京的声音”能迅速 传播到全国城乡 . 无怪乎近年来很多人在期盼“大喇 叭”的回归 ${ }^{[11]}$. 当然现在不能简单地采用当时的技术, 但其公众化应用的模式和受众广泛性及实时性的效 果很有借鉴意义.

既然数字广播终端产品在个性化电子产品的竞 争中不占优势，而公众化应用又在个性化浪潮中被 淡化, 那么数字广播可以把公众化应用作为其发展 定位，把主攻方向放在通信所不能为的领域，扬长避 短，获得独有的发展空间.广播利用通信产业的发展 势头成为其互补技术将能得到更大发展.

\section{3 发展我国数字广播的政策探讨}

同其他国家一样，我国广播数字化的初衷是为 了代替模拟广播. 但由于模拟广播已有的普及程度 以及国内模拟收音机太便宜, 要取代模拟广播需要 有政策上的支持. 英国推广 $\mathrm{DAB}$ 的做法是利用数字 
广播节省频率资源的优势, 在数字频段内增加节目 数. 目前伦敦地区可以收听到 50 多个 DAB 台, 而调 频 (frequency modulation, FM)广播只有 10 多个台. $\mathrm{DAB}$ 以其节目多、音色好逐渐受到人们的喜爱. 当 然在英国, DAB 收音机和模拟收音机的价格相差不 大也使得 $\mathrm{DAB}$ 收音机容易被接受. 欧洲国家推广 DAB 的另一个政策是在数字用户达到一定比例时, 提出模拟广播转换到数字广播的时间表，使得广播 行业和用户不再观望. 相比之下，我国尚未有具体的 支持广播数字化的政策. 北京 DAB 节目有 17 套, 但 其中 16 套都是重复的 FM 节目. 其实北京 DAB 信号 覆盖和节目接收效果都很好, 并且连续播放 8 年有余, 但这样一个大都市知道 DAB 的人零翏无几, 市场上 也几乎没有 $\mathrm{DAB}$ 接收机出售.

国内 DAB 运营单位也一直试图利用数字广播技 术特点创造新的应用 ${ }^{[12 ~ 14]}$, 但一直未能取得突破. 希望本文所提出的公众化应用方向, 可以扩展数字 广播业务范围, 并积极地影响我国政策/标准的制定 以及广播技术和产业的未来规划发展.

阻碍数字广播发展的另一个因素是自主标准问 题, 国内总有人担心 DAB 标准是舶来品. 我们首先 肯定自主标准的愿望和尝试是积极的, 但一个标准 是否有必要以及能否成功, 有各种因素影响 ${ }^{[15]}$. 如 果条件不具备, 一味以推出自主标准为目的, 反而会 影响技术和产业的发展.

以数字广播为例, 由 WorldDMB 推出的 Eureka 147 系列标准是国际上公认的比较先进和成熟的技 术标准系列, 已经为 50 多个国家所采用. 我国的 GY-T/214-2006 标准也是基于此系列. 因此在我国推 广这套标准是合理和经济的.

Eureka 147 系列标准本身不收费, 但其标准中所 采用的专利技术是收费的, 如 DAB+标准中的 SBR 技术要交纳专利费用. 避免专利费用的最有效方法 是技术创新. 我们 ${ }^{[16]}$ 在设计 $\mathrm{DAB}+$ 基带解码芯片时, 在按照标准做的同时, 提出了新的高频复制算法, 不 仅运算更简单，而且避开了专利问题.

有效利用国际标准和国外专利技术是明智的做 法. 把自主标准与自主创新等同起来, 甚至与“爱国 主义”联系，是片面的，并且有误导作用. 自主标准 不意味着完全避开专利费用, 因为许多关键技术仍 将不可避免地采用国外专利. 另一方面, 如果每个国 家或地区都要执行自己的“自主标准”，倒有闭关自
锁之嫌，就象 100 年前火车不同轨的情况.

基于上述认识，我们呼吁我国明确支持 Eureka 147 系列和我国的 GY-T/214-2006 标准, 尽快提出广 播数字化的时间表. 也正是基于上述认识, 我们课题 组近十年一直致力于 $\mathrm{DAB}$ 技术的研究, 致力于公众 化应用的研究与实践, 努力在标准框架内创新, 掌握 并发展核心技术，初步实现了适合中国国情的特色 应用。

\section{2 数字广播公众化应用}

数字广播公众化应用是以数字广播发射系统为 平台，权威发布公共信息，包括政务信息、天气、交 通、预警等与民生息息相关的信息. 与发射系统相对 应的是在公共区域广泛放置的多媒体接收终端和专 业人员配发的个人终端. 除了接收音频信号的喇叭 和收音机，更多使用液晶显示 (liquid crystal display, LCD)屏和发光二极管 (lighting emitting diode, LED) 屏等显示图片、文字和视频.

虽然其他媒体形式也可以作为公众化应用平台, 如商业广播电视、互联网、报纸杂志、手机等，但这 些平台都有一个共同问题一一用户选择权. 广播电视 用户可以选择其他娱乐节目，互联网上专业、严肃内 容的点击率反而低，手机短信过于简单，加之互联网 和手机短信上的垃圾内容过多, 紧急事件会让人半 信半疑. 另外, 技术上互联网和手机短信并不能及时 到达所有用户．因此这些平台虽然可以在一定条件 下起到公众化的作用, 但效果并不理想. 这也就是平 台多了，公共信息发布的效果反而不如以前“大喇 叭”的原因。

以数字广播为平台的公众化应用将由发射台控 制所有公共终端的开启, 受众没有选择权, 所播送的 内容均为当地或本单位重要的信息, 使之成为当地 或单位“新闻发言人”的角色，群众自然会予以关注. 严峻的治安形势需要管理机构有自己的话语权, 在 紧急情况发生时群众也需要聆听权威声音, 否则一 个谣言或恶作剧都可能造成大面积恐慌或事故. 数 字广播公共信息发布平台可以在关键时刻起到及时 发布正确信息和安抚人心的作用. 数字广播的公众 化应用体现了专业性、权威性、受众性(覆盖范围内 所有人)、可靠性和强制性(无用户选择权).广播的单 向性在公众化应用中成为了一个优点.

在公共区域大量放置 LCD 和 LED 屏，发布图文 
信息可以起到较好的宣传效果和美化环境的作用, 能吸引过往人群的眼球. 已经发现, 视觉效果好于听 觉效果(没有噪音污染). 精心选择的图片和精练的语 言可以把本地区、本单位最亮丽的方面展示出来, 就 像家庭客厅所放置的数码像框. 图 1 是我校一些办公 楼门厅的 LCD 屏幕所显示的经典图片.

除了作为政府和单位的公共信息发布的平台, 还可以开发专业化的应用, 如多媒体广播教学、预警 和应急指挥系统、旅游景区应急系统等. 我国是教育 大国、旅游大国，又是自然灾害多发地，这些应用都 可能在全国范围内推广, 具有广阔的市场. 我们认为, 以公众化应用为导向, 将能产生一个由数字广播技 术所支配的特色产业.

\section{1 数字多媒体广播教学应用}

在学校建立一个小功率数字广播发射台覆盖校 园，可播放教务、校务、宣传甚至娱乐节目. 这套校园 数字广播系统的建设将显著提高教务多媒体系统数 字化水平和效率，提供学生思想教育和娱乐的平台.

(i ) 数字多媒体广播教学系统的亮点和优势. 目前各学校都具备了有线广播或 FM 广播, 教室里也 都有网络(internet 或 wifi), 部分学校建了网络广播. 但这些技术都有其局限性. 模拟广播(有线或 FM)覆 盖范围广, 但业务单一(只能播放语音), 并且有线广 播需布线(现在布线和维修越来越困难), FM 广播干 扰越来越厉害; 网络(包括网络广播)的问题是不能保 证不中断, 特别是教室多了更容易断. 而采用数字技 术的校园广播既有模拟广播的优势，又有网络技术 多媒体的便利, 接收终端数在覆盖区域内没有限制, 即所谓的“一对全体”.

一台 DAB 发射设备可以播 10 套以上节目(FM只 能发一套节目), 可以播放各种视频和语音课程, 发 送图片和文字信息, 满足多媒体教学的需要. DAB 广 播终端可以分组, 每一个终端可以分配 ID 号, 进行 身份识别. 这样各个终端可以分别接收不同内容, 并 且无 ID 号的终端不可以使用, 有利于管理和保密. 一旦在上课和考试时发生紧急情况, DAB 系统可以 立即发布预警和应急指示, 提高学校的公共安全应 急能力.

综上所述, DAB 是理想的多媒体教学平台, 而且 $\mathrm{DAB}$ 的建设成本明显低于网络和通讯方案, 与 $\mathrm{FM}$ 广播相当, 但功能增加很多.

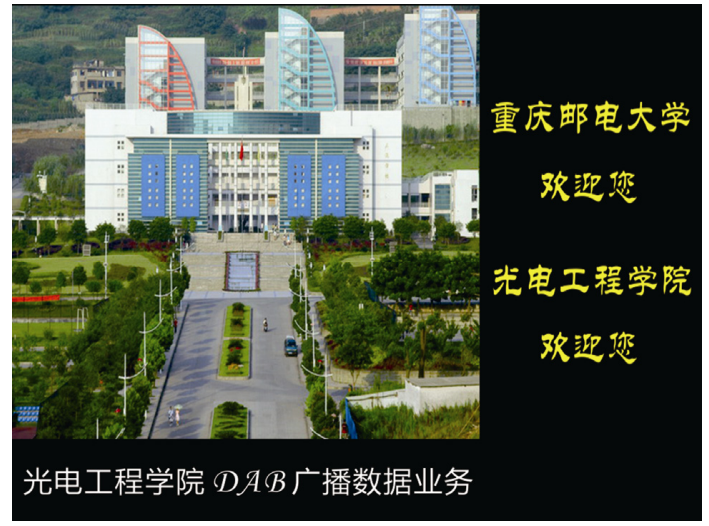

图 1 DAB 图文信息

(ii) 数字多媒体广播用于大型考试. $\mathrm{DAB}$ 非 常适合用于大型考试等重要场合. 播听力考试不仅 音质最好( $\mathrm{CD}$ 音色, 比有线广播或 $\mathrm{FM}$ 广播音色好), 而且内容可以编码, 保密性好. 除声音外, DAB 可以 播发视频、图片和文字, 按重庆市教育考试院要求, 达标考场必须全程播放考场信息提示，使用 $\mathrm{DAB}$ 的 图文形式发布最为简单可靠.

以高考和研究生人学考试为例, 通过这套系统 可以统一给每个教室发布语音和文字的考试信息. 在开考前 $30 \mathrm{~min}$ 开始, 发布语音的考试须知, 投影 仪或大屏幕则以文字和图片的形式同步显示考试须 知; 开考后语音必须停止, 而投影仪或大屏幕仍以文 字和图片的形式全程显示考试信息，包括当前时间、 所剩时间、指示(如请开始答题、可以交卷、请仔细 检查等内容, 图 2). 如有听力和视频考试内容, 则由 本系统统一播放. 这套系统使得考试更加规范.

(iii) 重庆邮电大学校园数字广播系统介绍. 重

\section{重庆市研究生入学考试}

考试科目：第一场

考试时问: $08: 30-11: 30$

现在时间: $09: 22$

考试备注: 无

离考试结束还有: 128 分钟!

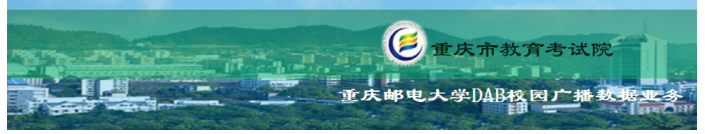

图 $2 \mathrm{DAB}$ 发布的考场提示信息 
庆邮电大学校园数字广播系统于 2013 年正式建设. 装备一套数字广播发射设备, 功率 $5 \mathrm{~W}$, 覆盖全校园. 终端主要是 500 个教室里的高质量音响(由 DAB 无线 接收)和带有 $\mathrm{DAB} U$ 口接收器的 PC 驱动投影仪, 视 频和文字内容可以通过投影仪播放, 室外喇叭为 $\mathrm{DAB}$ 无线接收的音柱(用于校园广播). 另有部分办 公楼门厅和教学楼门厅正安装 LCD 屏(采购 50 寸电 视屏), 通过 $\mathrm{DAB}$ 多功能终端, 接收和显示实时课 表、精品课程、名师风采、经典语录、教规训诫、教 务通知、教学成果展示等内容. 校园内已有的 LED 显示屏也将增加 DAB 无线接收功能.

这套校园数字广播系统对于提升教学条件档次 和教务管理能力起到很好的作用, 也减轻了技术支 持/维护的压力. 以 2014 年全国大学生英语竞赛为例, 有 4 个不同级别的听力考题需要同时播放, 通常是使 用 4 个播音控制器输出 4 路信号, 每路信号以有线方 式送到目标教室, 不仅管理复杂, 而且容易出错. 而 $\mathrm{DAB}$ 只需一套发射设备即可同时发送 4 路听力, 在 发射端通过软件操作即可控制每个考场的正确接收. 良好的效果得到重庆市教育考试院和重庆市教委技 术中心的好评和重视, 正与教委技术中心合作, 提出 建议方案供其他学校参考.

\section{2 预警和救灾}

用数字广播技术可以建立专业且专用的公共安 全信息发布和预警体系，可以在第一时间发布预警 信息到最大范围, 比其他所有的网络和媒介都快捷 和有效. 用数字广播技术可以在通信、交通和电力完 全中断的情况下，保持信息发布和命令下达的畅通， 实现有效救援指挥和灾区管理. 通过在地震多发区 乡镇配备便携式发射装置, 紧急情况下可以快速发 出求救信息, 甚至实现与外界的双向通信. 进一步, 全国地震系统(或应急办)可以建立应急救灾专用的 “广播应急通信”网络, 这样的网络可靠、不间断且可 以保证信息安全.

(i ) 数字多媒体广播预警系统. 基本的数字多 媒体广播系统由一套和多套发射设备与尽可能多的 接收终端组成(图 3). 发射设备由编码器、上变频器 和功放等组成. 发射功率 $1000 \mathrm{~W}$, 可以覆盖直径 80 $\mathrm{km}$ 范围. 终端设备包括 LCD 屏、 LED 文字显示屏和 智能喇叭等公共接收终端, 也包括带数字广播接收 功能的收音机、手机和电脑等个人终端.
公共场所的接收终端由政府配备, 准备一批移 动的 LCD 设备和喇叭以供临时放置. 各单位接收终 端自行购置, 可要求学校、医院、社区及人口密集单 位装配至少一台具有待机唤醒功能的终端, 可以在 紧急情况下自动开启高音喇叭. 并可逐步利用商店 门面的 LED 广告屏(平时商店正常使用, 应急信息优 先显示). 这些公共接收终端在灾害发生时可以覆盖 尽可能多的人群. 地震专业人员、消防队员、医务人 员和公务人员配备便携个人终端，接收专门信息和 指令. 每个发射台如能在信号范围内接收到相邻至 少一个发射台的信号, 那么它们就可以建立通信关 系, 各地具有通信关系的发射台就可以互联成网.

(ii) 快捷有效的预警和救灾指挥及应急通讯. 结合防灾预案事先准备好预警内容, 在灾害发生前 数天内进行防灾抗震科普知识介绍, 如疏散路线和 逃生知识, 使群众心理和物质上有所准备. 在灾害发 生前数小时可逐步加强预警的强度和级别. 在灾害 发生第一时间打开所有公共接收终端, 实现自动唤 醒和强制预警, 并形成声、光、电报警的强烈效果.

在灾后救援过程中, 发挥数字广播稳定工作和 覆盖范围广的优势, 可建成快捷有效的救援指挥体 系. 可以通过公共终端管理避难场所, 安抚群众, 也 可以实行交通管制, 通过 LED 引导和疏导救援车队. 所有救援人员可配备个人终端, 在上级部门的统一 指挥下实施有组织的救援. 在通信和交通完全中断 的极端情况下, 数字广播系统可以在上下级之间维 持联系和指挥. 上级机关可以通过数字广播系统向 下级单位下达指令和发送文件. 任何单位只要配备 有带数字广播接收装置的笔记本电脑并获得授权, 就可以及时获得上级机关的文件和文档.

重大灾害常常导致通信网和互联网中断, 造成灾 区和外界失去联系，给救援工作带来困难. 汶川地震 和雅安地震中, 最困难的就是联系不上的“孤岛”(如映 秀镇和宝兴县). 如何保障与这些“孤岛”的通信, 至今 仍缺少有效手段. 而数字广播在紧急情况下可以实现 应急通信. 只需在每个“孤岛”事先配备 DAB 发射机和 接收机, 就可以快速地发出求救的声音、图片和视频, 同时也可以接收外界应答和政府指示等信息.

(iii) 卫星与地面广播结合的 DAB 预警体系. DAB 除了可工作于适合地面广播的 III 波段(170 240 $\mathrm{MHz}$ )外，还可工作于适合卫星广播的 L 波段(1440 $1490 \mathrm{MHz})$.中国卫星通信集团公司从 2002 年起利 


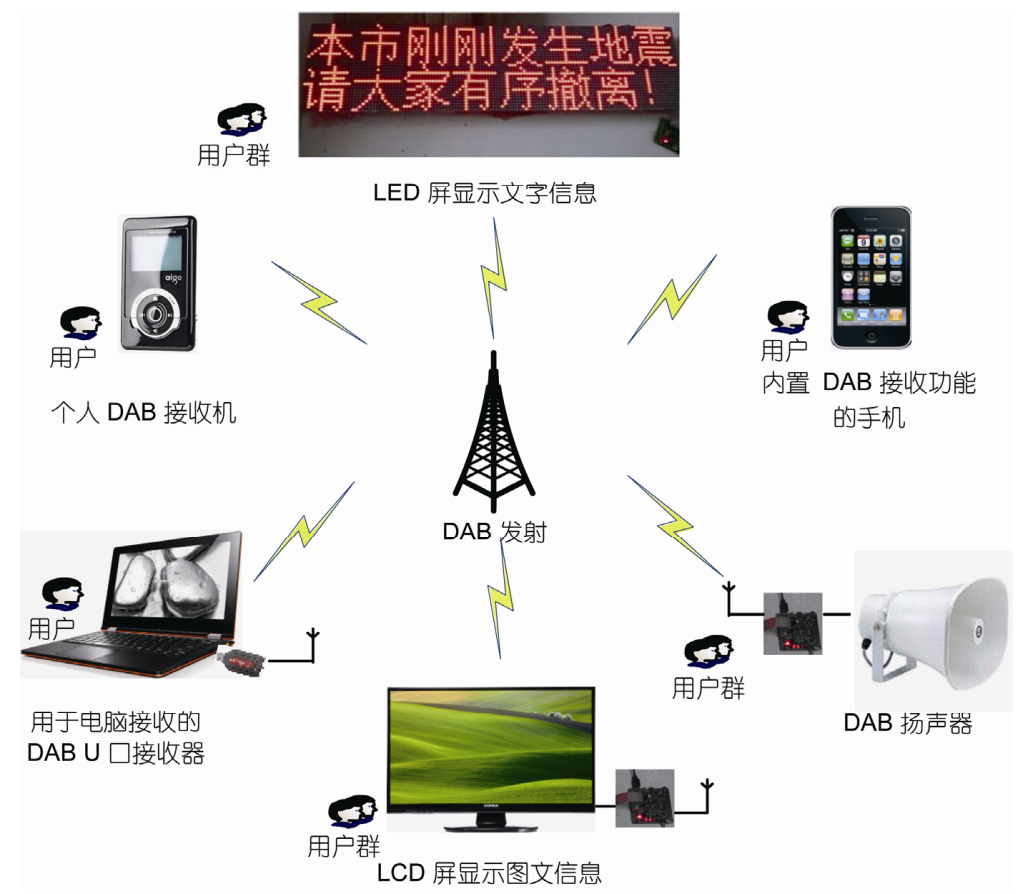

图 3 数字多媒体广播预警系统

用世广集团(Worldspace)的“亚洲之星”开展卫星 DAB 广播实验, 实现了气象信息广播和卫星应急指挥调 度等多种信息业务. 在此基础上, 2008 年国家气象局 提出了基于 $\mathrm{DAB}$ 的数字音频卫星广播预警系统 ${ }^{[17]}$. 在该系统中, 地方政府的预警信息通过专网逐级上 报后, 最终由国家预警信息发布平台上传给卫星发 射, 地面接收装置通过区域识别码进行选择接收. 该 系统具有覆盖面广的优点, 适合于对大区域以及偏 僻农村、荒漠或海岛等边远地区的预警信息发布.

但是卫星广播覆盖死角的问题比较突出, 对高 楼大厦林立的城市覆盖效果比较差. 卫星广播的预 警信息发布审批和启动过程比较长, 因此卫星广播 需要有地面广播作为补充. 地面广播对于局部地区 使用更为快捷便利, 具有更大地灵活性和实效性. 因 此, 以各个地区地面 DAB 预警系统为主, 结合全国 范围的卫星 DAB 大区域覆盖, 特别适合于我国疆域 辽阔、地理情况复杂、灾害分布不均的国情.

\section{3 景区公共安全应用}

近年来国内几个著名景区都发生过失控事故. 事故原因各不相同(游客众多、缆车停运、道路不通 等), 而造成失控局面的一个共性的问题是手机 “打 爆”了. 现在手机已成为人们日常生活交流最重要的
工具之一，当有突发事件时手机很容易“打爆”，而手 机 “打爆” 会使得游客因为互相联系不上而不知所措. 景区工作人员没有手机也不知如何解释和疏导, 景 区不能够发布信息, 因此不能够指挥, 任何应急预案 也实施不了, 最终引起大范围的恐慌和混乱. 如果配 备了数字广播应急系统, 紧急时所有终端全部开启, 反复播放语音和图文内容(如事故原因、处置告知、 游客注意事项、地图、撤离路线), 可以有效地安抚 游客, 避免恐慌. 景区员工通过个人终端接收指令, 各司其职, 有效疏导, 可以把事故控制在最小的范围. 此系统平时可用于宣传相应的旅游信息、景点特色 (文化和历史背景)和活动安排.

\section{3 我国在数字广播公众化应用方面的领先 地位}

DAB 国际标准 ${ }^{[1]}$ 和我国标准 ${ }^{[2]}$ 均支持广播节目 以外的多媒体内容, 使得数字广播公众化应用可以 在标准框架下实施, 为今后推广降低了门槛. 但是一 种新的应用也带来了新的技术挑战, 特别是要能够 主导这个产业，必须具有核心技术和优势.

目前国际市场上销售的 DAB 接收机大都是在我 国生产的, 我国又是 LCD 和 LED 生产基地, 因而接 收终端和显示器件的供应都可以立足于国内企业. 
我国在 DAB 领域已经有了自己的核心芯片, 在此基 础上开发了低成本 DAB 发射机, 核心芯片和整机均 具有自主知识产权. 我们在国际上率先提出公众化应 用方向, 重庆邮电大学实施了基于 DAB 数字广播技 术的多媒体教学系统, 是国际上第一个应用实例. 我 国已经具备了从核心芯片、整机技术到产业和应用的 完整技术优势, 使我国在数字广播公众化应用方面有 着比西方发达国家(DAB 标准发源地)更有利的条件.

(i ) 核心芯片. DAB 核心芯片主要包括 RF 芯 片和基带芯片, 在国内均有单位开发和生产, 其中不 乏大企业. 以我们与企业合作开发的低功耗 DAB 基 带芯片为例, 该芯片采用自主设计的可配置运算单 元, 用全硬件设计的方法, 将 DAB 的 COFDM 信道 解码和 MP2/AAC/AAC+信源解码全部集成在一起. 由于核心算法和低功耗设计方法的突破 ${ }^{[16]}$, 芯片面 积仅为国外同类芯片的 $1 / 4$, 功耗 $<26 \mathrm{~mW}$. 由于具有 自主技术, 这款芯片没有购买任何一个 IP 核, 也无 须交纳任何专利费.

(ii) 数字收音机. 目前国际市场上销售的 $\mathrm{DAB}$ 数字收音机大都是在我国生产, 但都是贴牌产 品(如 Pure, Robert 和 Bush 等), 只有一款是以我国知 名品牌爱国者(Aigo)在英国市场销售. 这款接收机采 用上述 $\mathrm{DAB}$ 基带芯片, 由作者自主设计, 重庆某家 企业加工生产, 具有完全自主产权. 通过整机的优化 设计和创新的分时控制方法 ${ }^{[18]}$, 该接收机在灵敏度、 功耗和稳定性等方面都优于国外品牌.

(iii) 低成本低功耗的 DAB 发射机. 目前商用 的 DAB 发射机都是国外生产, 体积大、成本高. 我 国一些城市开播 DAB 是从国外进口的设备, 价格在 数百万元左右, 不适合广泛应用. 我们 ${ }^{[19]}$ 在 DAB 的 编码算法和系统方面进行了优化, 设计的 DAB 发射 机仅需一台笔记本电脑、一块 COFDM 编码板和一个 功率放大器, 使得 DAB 发射机成本有 2 个数量级的 降低, 学校或乡镇单位均可以采用, 使公众化应用成 为可能. 新的系统方案也使得 DAB 发射机的功耗大 为降低, 在断电时可用直流电供电. 目前只有我国能 提供这样的低功耗便携式 DAB 发射机.

(iv) 多媒体智能终端. DAB 前期的研究主要 是音频和视频节目的传送与接收, 而公众化应用更 多的是图片、文字和文件的传送与显示, 这可通过

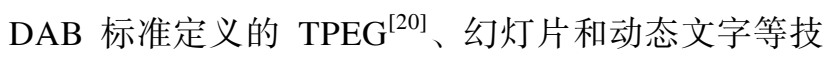
术来实现. 公共信息的多样化还对接收终端的智能
性提出了要求. 具体而言, 就是要求终端可以自动唤 醒并强制预警, 可以智能地从多路信息中选择需要 本机接收的信息并自动播放, 可以根据发射的指令 信息在声音、图像和文字等多种播放状态中自动切换, 可以智能判断信息的类型和级别并做出正确响应.

我们已开发了具有上述功能的发射系统和智能 接收终端. 发射端按照一定规则对公共信息进行编 码, 接收终端通过 DAB 解码和身份识别智能地判断 出需要接收的信息, 并通过智能控制技术驱动 LED 或 LCD 屏进行显示. 基于这些技术, 重庆邮电大学 实施了国际上第一个基于 DAB 数字广播技术的多媒 体教学系统, 已用于研究生人学考试、本科招生考 试、全国大学生英语考试和预警演练等有严格要求的 场合. 重庆邮电大学微电子工程重点实验室建立的 $\mathrm{DAB}$ 预警应急信息发布和通信系统, 解决了实际应 用所需要的技术难题.

(V) 国际影响力. 2013 年 10 月, 由国际组织 WorldDMB 和重庆邮电大学共同举办了 DMB 公共信 息发布、预警和应急通讯技术国际研讨会, 这是 WorldDMB 范围内的第一次这种类型的学术会议. 在这次会议上, 我们向国内外同行介绍并展示了以 上的研究成果和应用. 国际组织 WorldDMB 在其官 方网站 Newsletter 上报道了此次 DMB 国际研讨会 ${ }^{[21]}$, 突出了中国学者在研讨会上所作的主题报告, 对重 庆邮电大学率先利用 DMB 技术进行多媒体教学和预 警的创新实践给予了充分肯定和较高评价. 继在我 国首次成功举办此类专题研讨会之后, 亚太广播联 盟于 2014 年 6 月在印度尼西亚召开了以气候变化和 自然灾害预警救灾为主题的国际会议 (http://www. abu.org.my/ccdrr), 专门安排了关于数字广播技术的 主题报告. DAB 公众化应用的理念正在得到越来越 多的国家和机构的重视.

\section{4 结论}

我们认为数字广播的未来发展方向应从个性化 转向公众化, 从而根本性地改变广播形态, 由单一的 声音广播节目变为多媒体形态. 广播技术可以弥补 通讯和互联网技术的先天不足, 成为现代通信技术 的互补技术, 从而极大地提升广播技术的重要地位. 数字多媒体广播技术在公共信息发布和教育、预警救 灾和景区安全等专业领域的应用都可能在全国范围 内推广, 具有广阔的市场前景. 
我国在数字广播公众化应用方面，处于国际领 先水平，具备了从核心芯片、整机技术到产业和应用
的完整的技术优势. 以公众化应用为导向, 将能够在 我国产生一个由数字广播技术所支配的特色产业.

\title{
参考文献
}

1 European Broadcasting Union. ETSI EN 300 401, Radio broadcasting system; Digital audio broadcasting (DAB) to mobile, portable and fixed receivers. 2006

2 国家新闻出版广电总局. GY/T214-2006, 30MHz-3000MHz 地面数字音频广播系统技术规范. 2006

3 European Broadcasting Union, ETSI TS 102 563, Digital audio broadcasting (DAB); Transport of advanced audio coding (AAC) audio. 2007

4 European Broadcasting Union, ETSI TS 102 428, Digital audio broadcasting (DAB); DMB video service; User application specification. 2006

5 Lee J H, Lim J S, Lee S W, et al. Development of advanced terrestrial DMB system. IEEE Trans Broadcast, 2010, 56: 28-35

6 Lee G, Lee H, Yun K, et al. Implementation of 3D T-DMB receiver for three-dimensional data service. In: Oy C S, ed. 2010 Digest of Technical Papers, International Conference on Consumer Electronics (ICCE). Tampere Fenland. Piscataway USA: IEEE, 2010. 93-94

7 Lim Y, Kim K, Jeong J. Hybrid interactive data service architecture over T-DMB with mobile network. IEEE Trans Consum Electr, 2010, 56: $2826-2833$

8 You Y, Jung S, Kim D K. Integrated mobile terrestrial digital multimedia broadcasting player compliant with the T-DMB, CMMB, and ISDB-T standards. IEEE Trans Consum Electr, 2010, 56: 488-493

9 Yang R. Considerations and suggestions on improvement of communication network disaster countermeasures after the Wenchuan earthquake. IEEE Commun Mag, 2011, 49: 44-47

10 Becker S M. Learning from the 2011 great east Japan disaster: Insights from a special radiological emergency assistance mission. Biosec Bioterr Biod Strat Pract Sci, 2011, 9: 394-404

11 谭清波, 刘朝英. 气象预警大喇叭在农村的运用前景分析. 贵州气象, 2013, 37: 59-64

12 秦学刚. 推送式广播技术催生点播式广播服务. 中国广播, 2010, 7: 95-96

13 盛国芳, 高鹏, 吴智勇, 等. 基于 DAB 系统的非实时数据音频业务. 广播电视信息, 2011, 3: 88-90

14 曹桔香, 刘蕾. 北京电台推送式广播系统的研究与应用. 世界广播电视, 2013, 3: 100-101

15 王国裕, 李田, 陆明荣, 等. 数字广播 DAB 在英国的进展和在中国的机会与困境. 数字移动时代, 2009, 5: 38-41

16 Wang G, Zhang H, Lu M, et al. Low-cost low-power ASIC solution for both DAB+ and DAB audio decoding. IEEE Trans Large Scale Integr Sys, 2014, 22: 913-921

17 李春来，陈永涛，刘然. 卫星数字音频广播技术在灾害预警信息发布中的应用. 中国应急管理, 2008, 9: 50-53

18 Zhang H, Wang G, Lu M. A high performance and low power consumption USB DMB receiver. In: Feng J, ed. Proceedings of 2011 International Conference on Computer Science and Service System. Red Hook: Curran Associates Inc., 2011. 1931-1934

19 Lu M, Wang G. Means for implementing a DAB receiver channel decoder. GB2429611, 2010, UK

20 European Broadcasting Union. BPN 027-5, Transport protocol experts group (TPEG) specifications-Part 5: Public transport information application. 2002

21 Wang G Y. Summary of workshop on the public application of DMB at CQUPT in October 2013, http://worlddabeureka.org/2014/02/ 05/summary-of-workshop-on-the-public-application-of-dmb-at-cqupt-in-october-2013/2014-02-20

\section{Developmental trend of digital broadcasting in China}

\author{
WANG GuoYu, ZHANG HongSheng \& LU MingYing \\ Key Laboratory Microelectronic Engineering, Chongqing University of Posts and Telecommunications, Chongqing 400065, China
}

This paper analyses the difficulties and opportunities for Chinese digital broadcasting development and suggests the shift from traditional radio programs to public applications. Digital broadcasting has inherent advantages in this direction and can be complement to modern communication techniques. And thus has potential to open up a massive new DAB-dominant market. Applications of DAB on campus and DAB for public safety are reported as examples, with key techniques introduced.

digital broadcasting, digital audio broadcasting (DAB), digital multimedia broadcasting (DMB), public application, alarm, emergency warning, public safety

doi: 10.1360/N972014-00081 